\title{
Measuring Apoptotic Cell Engulfment (Efferocytosis) Efficiency
}

\author{
Matthew C. Gage ${ }^{*}$
}

${ }^{1}$ Centre for Clinical Pharmacology, Division of Medicine, University College of London, 5 University Street, London, WC1E 6JF, United Kingdom.

"Corresponding author:

Matthew C. Gage, Centre for Clinical Pharmacology, Division of Medicine, University College London, London WC1E 6JF, UK. Phone: +44 (0)20 76796535.

E-mail:m.gage@ucl.ac.uk

\section{Running Head}

Macrophage efferocytosis assay

\section{Abstract}

Efferocytosis is the process of recognising and removing dead and dying cells, performed by a variety of phagocytic cells including macrophages. It has recently been shown that Liver $X$ Receptor (LXR) signalling in macrophages regulates the expression of important efferocytosis receptors, bridging and signalling molecules. Here we describe a sensitive yet robust efferocytosis assay, optimised to measure bone marrow derived macrophage (BMDM) apoptotic cell engulfment capability. This assay can be applied to genetically or pharmacologically altered BMDMs.

\section{Key words}

Nuclear receptors, efferocytosis, apoptosis, macrophages, BMDM, Jurkats, engulfment. 


\section{Introduction}

Efferocytosis is the term given to the process of the recognising, engulfing and processing dead and dying cells (1) which is performed by a variety of phagocytes including macrophages (2). Macrophages are a heterogeneous population of phagocytotic leukocytes (3) which have high efferocytotic capability (4), shown to engulf dead and dying cells in healthy tissues with high cell turnover such as the bone marrow, spleen and thymus (5). Macrophages also perform their efferocytic duties in atherosclerosis (6) - the leading cause of cardiovascular disease (7) - where macrophage efferocytosis capacity in atherosclerotic plaques has been linked to the progression of necrotic cores through, among others, the tyrosine kinase receptor Mer (MerTK) (6). Macrophages express nuclear receptors, several of which have been linked to efferocytosis activity including PPARy, PPARס, RXRa (8) and Liver X Receptors (LXRs) (9). LXRs regulate the expression of the important efferocytosis receptor MerTK (10), and several other bridging and signalling molecules (11) directly involved in efferocytosis.

This protocol is based upon original methodology described by Marissa Nadolski and Ed Thorp (12), and has been optimised to provide a simple yet robust assay for measuring efferocytosis efficiency in a relatively small number of cells. This is an ideal feature of an assay, when the subject is a precious commodity such as primary bone marrow derived macrophages from a rare genetically modified mouse (13), with which you may also want to perform additional experiments with. In this chapter the utilisation of 8 well chamber slides is described, which allows for multiple conditions to be tested such as various time points and a range of agonists and concentrations. After addition of Jurkat cells undergoing apoptosis, the BMDMs are fixed, imaged, and the efficiency of efferocytosis is quantified using freely available software.

\section{Materials}


Prepare all solutions using ultrapure water and analytical grade reagents. Prepare and store all reagents at $4{ }^{\circ} \mathrm{C}$ or $-20{ }^{\circ} \mathrm{C}$ as indicated (unless otherwise noted). Follow all local waste disposal regulations when disposing of waste materials.

\subsection{Jurkat cell culture}

1. Glutamine: $200 \mathrm{nM}$

2. Gentamycin: $10 \mathrm{mg} / \mathrm{mL}$

3. Complete RPMI media: (RPMI, $10 \%$ FBS, $1 \%$ glutamine, gentamycin 20 $\mu \mathrm{g} / \mathrm{mL})$.

4. Jurkat cells (see Note 1 and 2).

5. $T 25 \mathrm{~cm}^{2}$ filter cap flasks

6. Sterile polystyrene pipettes ( $5 \mathrm{~mL}, 10 \mathrm{~mL}, 25 \mathrm{~mL})$.

7. $70 \%$ ethanol.

8. Cell incubator with programmable control of $\mathrm{CO}_{2}$ and temperature.

\subsection{Bone marrow derived macrophage cell culture}

1. Murine bone marrow derived macrophages (see Note 3).

2. Cell scrapers.

3. Sterile 8 well culture slides.

4. Cell incubator with programmable control of $\mathrm{CO}_{2}$ and temperature.

\subsection{Preparation of apoptotic cells}

1. Complete RPMI media: (RPMI, $10 \%$ FBS, $1 \%$ glutamine, gentamycin 20 $\mu \mathrm{g} / \mathrm{mL})$.

2. $10 \mathrm{~cm}$ sterile TC dish.

3. Calcein $\mathrm{AM}(1 \mathrm{mg} / \mathrm{mL}$ in $\mathrm{DMSO})$ store at $-20^{\circ} \mathrm{C}$.

4. Warmed PBS $1 \times 37^{\circ} \mathrm{C}$.

5. UV irradiator. 
6. Sterile polystyrene pipettes $(5 \mathrm{~mL}, 10 \mathrm{~mL}, 25 \mathrm{~mL})$.

7. $70 \%$ ethanol.

8. Cell incubator with programmable control of $\mathrm{CO}_{2}$ and temperature.

9. $1 \%$ paraformaldehyde.

\subsection{Efferocytosis assay}

1. Complete DMEM: (DMEM, $10 \%$ FBS, $1 \%$ glutamine, gentamycin $20 \mu \mathrm{g} / \mathrm{mL}$ ).

2. Apoptosing Jurkat cell solution (from step 2.3).

3. BMDM cultured on 8 well culture slides

4. Ice cold PBS $1 \mathrm{X}$

5. $1 \%$ paraformaldehyde

6. $70 \%$ ethanol.

7. Single channel manual pipettes $(1 \mathrm{~mL}, 200 \mu \mathrm{L})$,

8. Cell incubator with programmable control of $\mathrm{CO}_{2}$ and temperature.

\subsection{Slide preparation}

1. 8 well culture slide removal tool (see Note 4)

2. Fluoromount aqueous mounting media (Sigma).

3. $22 \times 50 \mathrm{~mm}$ glass cover slips

4. Nail varnish.

\subsection{Image acquisition}

1. Fluorescent microscope with 20x magnification and GFP filter (excitation wavelength $450-490 \mathrm{~nm}$, emission wavelength 515-565 nm) and digital camera (see Note 5).

2. Image acquisition software (see Note 6).

\subsection{Efferocytosis quantification}


1. ImageJ image analysis software with grid overlay plugin (https://imagej.nih.gov/ij/download.html).

\section{Methods}

\subsection{Jurkat cell culture}

This is your source of apoptotic cells.

1. Grow Jurkat cells in complete RPMI in $T 25 \mathrm{~cm}^{2}$ filter cap flasks. Count daily with a hemocytometer maintaining cell density at between $2 \times 10^{5}$ and $1 \times 10^{6}$ cells $/ \mathrm{mL}$ (see Note 7).

2. Grow sufficient number of cells as required for the number of experiments you are performing.

\subsection{BMDM cell culture}

1. When BMDM reached $90 \%$ confluency (see Note 8 ), scrape the cells from the TC dish and pipette up and down several times in a $10 \mathrm{~mL}$ pipette to homogenise the cells into a single cell suspension.

2. Count BMDMs on a hemocytometer

3. Centrifuge cells at $500 x g$ for $10 \mathrm{~min}$.

4. Resuspend cell pellet in complete DMEM to a final concentration of $2.5 \times 10^{5}$ cells $/ \mathrm{mL}$.

5. Seed re-suspended BMDM in onto 8 well slides, $250 \mu \mathrm{L} /$ well (see Note 9 and Fig. 1).

6. Incubate overnight at $37^{\circ} \mathrm{C}, 5 \% \mathrm{CO}_{2}$.

\subsection{Preparation of apoptotic Jurkat cells}


1. Count Jurkat cells using a hemocytometer.

2. Centrifuge cells at $500 x g$ for $5 \mathrm{~min}$.

3. Resuspend pellet at $2 \times 10^{6} \mathrm{cells} / \mathrm{mL}$ and plate $20 \times 10^{6}$ cells $(10 \mathrm{~mL})$ onto a $10 \mathrm{~cm}$ TC dish.

4. Add $10 \mathrm{uL}$ of Calcein AM solution to the dish and mix thoroughly by gently pipetting up and down several times using a $10 \mathrm{~mL}$ pipette.

5. Incubate for approximately 2 hours at $37^{\circ} \mathrm{C} 5 \% \mathrm{CO}_{2}$ (see Note 10 and Note 11).

6. Collect cells in $15 \mathrm{~mL}$ conical tube.

7. Wash dish with $3 \mathrm{~mL}$ of Jurkat complete media and add to the conical tube of Jurkat cells.

8. Centrifuge at $500 x g$ for 5 min (see Note 12 and Fig. 2).

9. Wash cells by removing the supernatant, resuspending the cells pellet in $5 \mathrm{~mL}$ of warmed PBS $1 \mathrm{X}$ and centrifuging at $500 \mathrm{xg}$ for $5 \mathrm{~min}$.

10. Repeat step 9 twice more for a total of three PBS washes.

11. Resuspend the cell pellet in $10 \mathrm{~mL}$ of warmed $\left(37^{\circ} \mathrm{C}\right)$ Jurkat complete media and plate onto a $10 \mathrm{~cm}$ TC dish.

12. Irradiate cells for 5 minutes (see Note 13 and 14). Swirl dish every 60 seconds to ensure homogenous exposure of cells to UV light source.

13. Incubate the irradiated cells at $37^{\circ} \mathrm{C}$ for approximately 2 hours (see Note 15 ) until approximately $50 \%$ of the cells are visibly blebbing (Fig. 3 ).

14. Collect the cells into a $15 \mathrm{~mL}$ conical tube.

15. Centrifuge at $500 x g$ for $5 \mathrm{~min}$. Resuspend the apoptotic cells at $2.5 \times 10^{5} \mathrm{cells} / \mathrm{mL}$ in complete DMEM media.

\subsection{Efferocytosis assay}

1. Remove complete DMEM media from chamber well slides with BMDM.

2. Add apoptotic Jurkats in a 1:1 ratio to the BMDM $(250 \mu \mathrm{L})$. 
3. Incubate BMDM and apoptotic Jurkats together for various periods of time ranging from 0 to $90 \mathrm{~min}$ (see Note 16).

4. Remove media, and immediately wash cells with $250 \mu \mathrm{L}$ ice cold PBS.

5. Remove PBS and repeat ice cold PBS washes twice more for a total of 3 washes (see Note 17).

6. Add $250 \mathrm{uL}$ cold $1 \%$ PFA solution and incubate at $4^{\circ} \mathrm{C}$ in the dark for $10 \mathrm{~min}$.

7. Repeat one more PBS wash step.

8. Add $250 \mu \mathrm{L}$ PBS to cover each well. Slides can now be stored wet overnight at 4 ${ }^{\circ} \mathrm{C}$ in the dark or proceed immediately to slide preparation.

\subsection{Slide preparation}

1. Flick off PBS from the 8 well slides.

2. Remove the 8 well plastic frame using the supplied removal device in accordance with manufacturer's instructions.

3. Add 3-4 drops of mounting media along the length of the slide.

4. Carefully place glass coverslip onto the slide, taking care not to introduce bubbles (see Note 18).

5. Gently brush a small amount of nail varnish on to the edges of the slide to seal it (see Note 19).

6. Allow varnished slides to dry in the dark at room temperature for a minimum of 1 hour (see Note 20).

\subsection{Image acquisition}

1. At the microscope, image slides at $20 x$ magnification using the GFP filter in a darkened room. 
2. Acquire a minimum of 3 images per well to ensure fair representation of the sample (see Note 21).

\subsection{Efferocytosis quantification}

1. Open image using ImageJ software on appropriate computer (see Note 22).

2. Divide images into 9 equal squares with grid overlay plugin as shown in Fig. 4.

3. Count the total number of cells (both the bright and dark adherent BMDMs) in a grid square as shown in Fig. 4.

4. Count the number of (1) bright fluorescent cells (which have engulfed labelled Jurkats) in the same grid square as shown in Fig. 5a and b.

5. Count the number of BMDM cells with attached Jurkats (2) in the same grid square as shown in Fig. $5 a$ and b.

6. Calculate the proportion of total cells performing efferocytosis (efferocytosis efficiency) as \% of total cells by using the following equation:

(Total number of cells / (Number of bright cells + number of cells with attached Jurkats)) $\times 100$.

7. Repeat for the remaining 4 grids as shown in Fig. 4 .

8. Calculate the mean efferocytosis efficiency (\%) for this image by adding the five resulting percentages together and dividing by 5 .

9. Repeat this for each of the 3 images you have for each well (see Note 23).

\section{Notes}

1. Jurkats are an immortalised $\mathrm{T}$ lymphocyte cell line which are widely available commercially. Follow supplier's instructions for storage and revival.

2. We have also applied this same apoptosis protocol to BMDMs to investigate the phagocytotic effects of BMDM capability on macrophages (BMDM), in addition to the Jurkat $\mathrm{T}$ cell model.

3. BMDM are a common resource. For isolation and culture and storage of BMDM see reference 13: 'Isolation, Culture and Polarisation of Murine Bone Marrow and Peritoneal 
Macrophages. Pineda-Torra I, Gage M, Juan A, Pello OM. Methods Mol Biol. 2015;1339:101-9.

4. The 8 well culture slide removal tool should be supplied by the manufacturer with the 8 well culture slides.

5. We use a Zeiss Axio Vert.A1 fluorescent microscope with a Zeiss Axiocam 503 mono digital camera with Zen Pro acquisition software.

6. We use the freely available Zen lite v2.3 software for viewing our acquired images on different hardware which is available from www.zeiss.com/microscopy/int/products/microscope-sofware/zen-lite.

7. Jurkats were counted by staining with Trypan Blue; 1 in 2 dilution. Using the culture conditions described, Jurkat cells were diluted in fresh media 1 in 5 , every 2 days to maintain a maximum cell density of $1 \times 10^{6}$ cells $/ \mathrm{mL}$ in T25 flasks standing upright.

8. Using our complete DMEM growth media, this was typically on day 5.

9. Pharmacological treatment can be applied at this point (for example an LXR agonist).

10. This incubation is to ensure maximum incorporation of Calcein AM dye.

11. Avoid bright light when using the Calcein AM (for example by switching off tissue culture hood light and covering cells in aluminium foil during transit) to preserve fluorescence.

12. Cell pellet should appear green (Fig 2), demonstrating incorporation of dye into living cells.

13. For irradiation we use a Hug Flight UV steriliser (model HF-15151-E). If using different equipment please follow your local health and safety regulations for UV exposure protection.

14. Remove lid of TC dish so cells get full UV exposure.

15. Check cells every 30 minutes with a light microscope for blebbing, as the rate of apoptosis induction may depend on the power of your UV lamp and distance of cells from UV source.

16. Efferocytosis efficiency may be influenced by your mutation/experimental condition - we have found it best to try a range of time points to establish $20-30 \%$ baseline 
efferocytosis, and saturation at approximately $80 \%$-which may not necessarily be solely measuring first-time uptake events.

17. We have found that if you are using multiple conditions and/or timepoints it helps to have more than one experimentalist on hand to ensure the incubations and washes are performed consistently in a timely fashion.

18. Using forceps, place coverslip gently from one end so that any bubbles that may form can travel through the mounting fluid, escaping to the edge and don't become trapped.

19. Take care not to move the glass cover slip when applying the varnish, as not to dislodge the fixed cells.

20. For best results we image our slides within 24 hrs of fixation.

21. See Fig. 1 for our typical slide layout and depiction of image acquisition.

22. There are other commercially available software packages for image analysis which may also be able to quantify your efferocytosis images. However, ImageJ is freely available and ensures maximum usability and audience for this protocol.

23. Our image analysis is performed blindly by our investigators, and the data is grouped after efferocytosis efficiency has been analysed for each image.

\section{Acknowledgements}

This work was supported by grants from British Heart Foundation Project Grant PG/13/10/30000 (IPT) and PG/16/87/32492 (MG). I would like to thank Ed Thorp for his insights into efferocytosis while optimising this assay, and Yu Zhang for image acquisition, used in figures 4 and 5, and Inés Pineda-Torra for equipment use and critical revision of the manuscript.

\section{References}

1. deCathelineau AM and Henson PM (2003) The final step in programmed cell death: phagocytes carry apoptotic cells to the grave. Essays Biochem 39:105-17

2. Sather S, Kenyon KD, Lefkowitz JB, et al (2007) A soluble form of the Mer receptor tyrosine kinase inhibits macrophage clearance of apoptotic cells and platelet 
aggregation. Blood 109

3. McNelis JC and Olefsky JM (2014) Macrophages, Immunity, and Metabolic Disease. Immunity 41:36-48

4. Li Y, Gerbod-Giannone M-C, Seitz H, et al (2006) Cholesterol-induced apoptotic macrophages elicit an inflammatory response in phagocytes, which is partially attenuated by the Mer receptor. J Biol Chem 281:6707-17

5. Kojima Y, Weissman IL, and Leeper NJ (2017) The Role of Efferocytosis in Atherosclerosis. Circulation 135:476-489

6. Thorp E, Cui D, Schrijvers DM, et al (2008) Mertk receptor mutation reduces efferocytosis efficiency and promotes apoptotic cell accumulation and plaque necrosis in atherosclerotic lesions of apoe-/- mice. Arterioscler Thromb Vasc Biol 28:1421-8

7. Mendis S, Puska P, and Norrving B (2011) Global Atlas on Cardiovascular Disease Prevention and Control. World Heal Organ Collab with World Hear Fed World Stroke Organ 155

8. Korns D, Frasch SC, Fernandez-Boyanapalli R, et al (2011) Modulation of macrophage efferocytosis in inflammation. Front Immunol 2:57

9. Edwards PA, Kast HR, and Anisfeld AM (2002) BAREing it all: the adoption of LXR and FXR and their roles in lipid homeostasis. $J$ Lipid Res 43:2-12

10. A-Gonzalez N, Bensinger SJ, Hong C, et al (2009) Apoptotic cells promote their own clearance and immune tolerance through activation of the nuclear receptor $L X R$. Immunity 31:245-58

11. Gage MC, Bécares N, Louie R, et al (2018) Disrupting LXRa phosphorylation promotes FoxM1 expression and modulates atherosclerosis by inducing macrophage proliferation. Proc Natl Acad Sci 201721245

12. Nadolski M and Thorp E (2009), Standardized Tabas Laboratory In-Vitro Efferocytosis Engulfment Assay, http://www.tabaslab.com/protocols/

13. Pineda-Torra I, Gage M, Juan A de, et al (2015) Isolation, Culture, and Polarization of Murine Bone Marrow-Derived and Peritoneal Macrophages. Methods Mol Biol 1339:101-9 